\title{
The study of protein composition of Triaenophorus sp. at different stages of the life cycle and in different body segments
}

\author{
A. Kochneva ${ }^{1 *}$, E. Borvinskaya ${ }^{1,2}$, D. Bedulina ${ }^{2}$ \\ ${ }^{1}$ Institute of Biology of the Karelian Research Centre of the Russian Academy of Sciences, \\ Petrozavodsk, Russia \\ ${ }^{2}$ Research Institute of Biology of Irkutsk State University, Irkutsk, Russia \\ *e-mail:kochnevaalbina@gmail.com
}

Key words: Cestoda, Triaenophorus sp., proteomics, stages of the life cycle, different body segments

\begin{abstract}
Motivation and Aim: Members of class Cestoda are unique organisms, which are obligate parasites with a complex life cycle that includes the life stages at very different habitats. Thus, cestodes can be considered as an appropriate model for the study of molecular adaptations. It is known that the tapeworms body structure is simplified, but a pronounced functional differentiation exists along the anteroposterior axis of the body and of the helminth strobila. The aim of this research was to investigate a protein polymorphism in different body segments (scolex, immature and mature segments of the strobila) and life stages of two species of Cestoda - Triaenophorus nodulosus (Pallas, 1781) and T. crassus (Forel, 1868).
\end{abstract}

Methods and Algorithms: The 2D-DIGE (two-dimensional difference gel electrophoresis) was used to study the electrophoretic mobility of proteins. A comparative analysis of gel images was carried out using the SameSpots software (TotalLab, England). Protein identification was performed with mass spectrometry (Agilent ESI-Q-TOF 6538 UHD (Agilent Technologies)) combined with HPLC (Agilent 1260 (Agilent Technologies)).

Results: Comparative analysis of 2D-DIGE electrophoregrams of plerocercoid and adult worms of $T$. crassus revealed two protein spots, significantly different in intensities. In T. nodulosus, significant differences were found for three protein spots. Significant differences in spot intensities were found in 2D-DIGE protein patterns of scolex, immature and mature segments of Triaenophorus sp. Two of these spots were identified as tropomyosin. Intensities of three spots were found significantly different on 2D-DIGE gels of plerocercoids of $T$. nodulosus and T. crassus. Protein patterns of adult worms differed in two spot intensities.

Conclusion: Thus, for the first time the electrophoretic protein mobility of two fish parasites species were described. This data will be useful in future research to develop species-specific antibody for helminthosis diagnostics in fishery.

Acknowledgements: Supported by the Russian Foundation for Basic Research (No. 17-04-01700_A). 